\title{
A suggestive evaluation and review on the synergy of Renin Angiotensin Aldosterone System (RAAS) and Immune response towards Covid-19
}

\author{
Abba Mohammed ${ }^{* 1}$, M.I. Shago ${ }^{1}$, G. Suleiman ${ }^{2}$ and M.U. Nasir ${ }^{2}$ \\ ${ }^{1}$ Department of Biochemistry, Yobe State University, Damaturu, Nigeria \\ ${ }^{2}$ Department of Chemistry, Yobe State University, Damaturu, Nigeria
}

\begin{abstract}
Globally, a "pause mode" to several activities has choicelessly been opted "on" to some extent as Covid-19 has bedeviled the life of humanity since December 2019. As a result, economical, educational, sociological and lots of fundamental paradigms were all inaccessible. Angiotensin Converting Enzyme 2 (ACE 2) paving a way to the incorporation of the SARS-CoV-2 by receptor mediation into the cells has to be scientifically focused towards an inoculative approach to synergically scotch the aiding capacity as well, within all the complications. The inoculation is dared to further boost the immunological responses towards the immunopathological fight against the slipped virus if at all the $\mathrm{CoV}-2$ adversely escaped to adapt on the receptors of the ACE 2. However, all these is as a result so far that the natural innate and the adaptive immune systems are finding it quite tough to hastily invade the pathogens and defend the human body system against the Covid-19 in some patients. Meanwhile, ingesting food of high great value of supplements in upshooting immune system as well as ensuring physical fitness and environmental hygiene are paramount to adherence in this trying moments and indeed all the time, as the world is looking forward to unveiling the veritable vaccine and outright therapeutics against the Corona virus.
\end{abstract}

Key words:- Severe Acute Respiratory Syndrome-Corona Virus2, Angiotensin Converting Enzyme 2, Immunopathological.

\section{INTRODUCTION}

The world is traumatizing and humans are dying. 1 According to World Health Organization (WHO), as of 16th August 2020, more than 21.5 million people have been affected with coronavirus and about 771,000 people have died worldwide. The world experienced coronavirus for the first time in 2002-2003 by Severe Acute Respiratory Syndrome (SARS) and in 2011 by Middle East Respiratory Syndrome (MERS). The causative agents for both cases (SARS-CoV and MERS-CoV, respectively) were newly identified coronavirus with zoonotic origin in the genus Beta coronavirus (Eakachai et al., 2020). The present corona virus (SARS-CoV-2) COVID-19 appeared for the first time in Wuhan, China, at the end of 2019. People are being affected by human to human transmission due to close contact (Yan-Rong et al., 2020; Guan et al., 2020). People affected by COVID-19 suffer from severe respiratory illness (Guan et al., 2020). People who are elderly and have numerous comorbidities are the most vulnerable to this virus (Kwok et al., 2020).
Not all people exposed to SARS-CoV-2 are infected and not all infected patients develop severe respiratory illness. Accordingly, SARS-CoV-2 infection can be roughly divided into three stages: stage I, an asymptomatic incubation period with or without detectable virus; stage II, non-severe symptomatic period with the presence of virus; stage III, severe respiratory symptomatic stage with high viral load (Wang et al., 2020). From the point of view of prevention, individuals at stage $\mathrm{I}$, the stealth carriers, are the least manageable because, at least on some occasions, they spread the virus unknowingly: indeed, the first asymptomatic transmission has been reported in Germany (Rothe et al., 2020). The role of asymptomatic SARS-CoV-2 infected individuals in disseminating the infection remains to be defined.

In the early months of this year, among over 1000 patients analyzed in Wuhan, except occasionally in children and adolescence, it infects all the other age groups evenly. About $15 \%$ of the confirmed cases progress to the severe phase, although there is a higher chance for patients over 65 to progress into the severe phase (Guan et al., 2020). One of the biggest unanswered questions is why some develop severe disease, whilst others do not? Clearly, the conventional wisdom based on overall immunity of the infected patients cannot explain this broad spectrum in disease presentation.

Moreover, the ongoing pandemic has stimulated study of the Renin Angiotensin Aldosterone System (RAAS), and how it can be manipulated to treat COVID-19. Researchers are examining whether drugs that act on the RAAS system might be useful to treat COVID-19. Possible benefits against COVID-19 are as yet purely theoretical, but pharmacological intervention may possibly be useful to synergically reduce binding of COVID-19 to cells and counteract the RAAS proinflammatory cascade if only there is at least little slip of the virus into the cell.

Like SARS-CoV-1, CoV-2 virus binds to the ACE2 enzyme through its trimeric spike glycoprotein and uses ACE2 to enter cells (Walls et al., 2020) and can target ACE2 in various cell types. 


\section{THE RENIN ANGIOTENSIN ALDOSTERONE SYSTEM (RAAS)}

As the depiction on the diagram in Fig. 1, the large polypeptide precursor angiotensinogen is converted by the enzyme renin to the decapeptide angiotensin I (Ang I).. Ang I is converted to Ang II by the enzyme (Angiotensin Converting Enzyme 1) ACE1 (often called simply ACE), cleaving the 2 C-terminal amino acids to produce the octopeptide Ang II Ang II is thus the major ACE1 product and the most active hormone in the system, acting in a pro-inflammatory manner, involving especially AT1-receptors. Ang II also acts the zona glomerulosa of the adrenal cortex to release aldosterone. Aldosterone itself has pro-inflammatory actions and causes sodium retention in the renal tubules, both involving mineralocorticoid receptors (MCR).

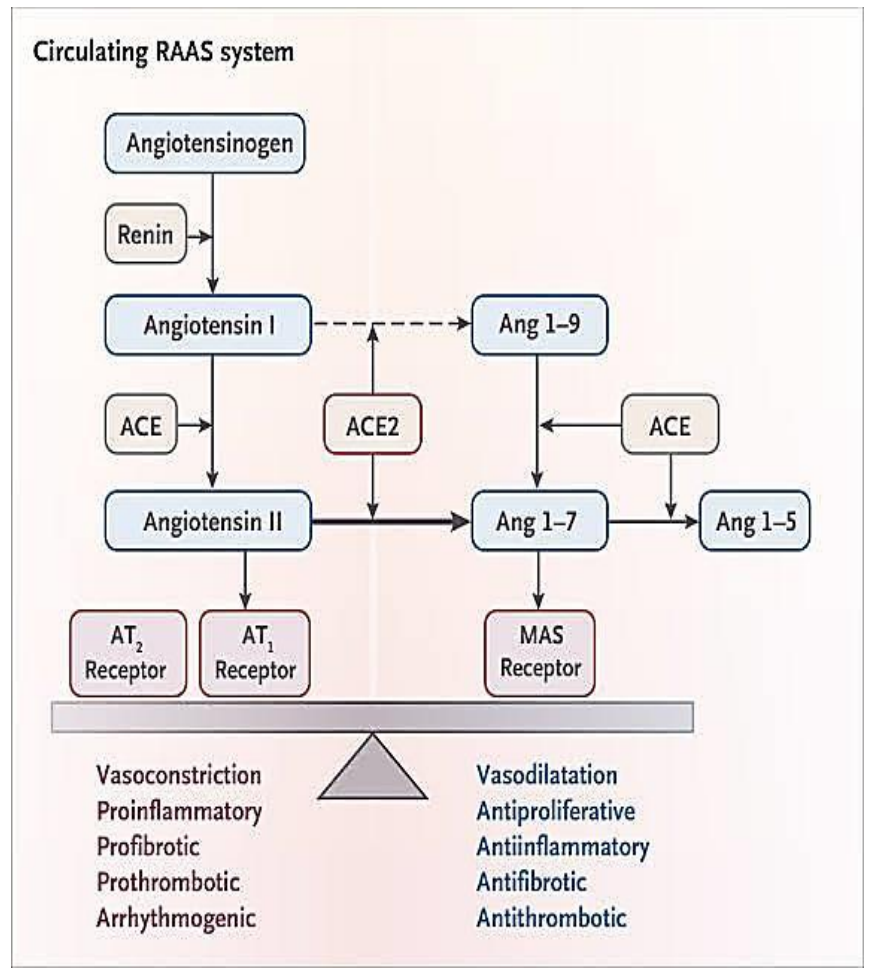

Fig. 1 illustrates the complex interactions in the RAAS.

Ang II acts at AT1-receptors (usually pro-inflammatory) and AT2-receptors (usually anti-inflammatory). The AT1-receptor has a wide distribution in the body, but the AT2-receptor is more localized (De Gasparo et al., 2000). In human lung, AT1-receptors were reported in the vascular smooth muscle and endothelium, macrophages and fibroblasts, whereas AT2receptors were found in the epithelium, and the proportion of AT1-receptors has been reported to increase in lung disease
(Bullock et al., 2001). Ang III and Ang IV are produced by the action of aminopeptidases to cleave the $\mathrm{N}$-terminal amino acids forming Ang(2-8) (Ang III) and Ang (3-8) (Ang IV). Ang IV may act mainly on AT4-receptors. However, both Ang I and Ang II can be converted to alternative products by the enzyme ACE2. The decapeptide Ang I is converted to the nonapeptide Ang(1-9) and the octapeptide Ang II is converted to the heptapeptide Ang(1-7), both by ACE-2 by cleaving the C-terminal amino acid. ACE1 converts Ang(1-9) to Ang(17), again cleaving $2 \mathrm{C}$-terminal amino acids. ACE-1 can also cleave a further 2 amino acids resulting in $\operatorname{Ang}(1-5)$. The major active agent formed involving ACE2 is thought to be Ang (1-7). Ang(1-7) has an anti-inflammatory profile of action.

\section{THE HUMAN SYSTEM'S SUSCEPTIBILITY TO THE HIGHLY TRANSMISSIBLE COV-2}

The crown-like new human-infecting SARS-CoV-2 is a positive-sense single-stranded RNA-enveloped virus with cave of ridges belonging to $\mathrm{CoV}$ family (Lu et al., 2020). Among the six CoVs pathogenic to humans, four of them have been associated with mild respiratory symptoms, Corman et al., (2019) while two of them, SARS-CoV and MERS-CoV caused severe respiratory diseases in affected individuals during their epidemic outbreak (Corman et al., 2019). SARSCoV-2 is the seventh identified CoV and, after SARS-CoV and MERS-CoV, the third zoonotic virus of CoVs that has been transmitted from animals to humans via an intermediate mammalian host (Chan et al., 2015; Mackenzie \& Smith, 2020) In particular, based on genetic analysis, Chinese horseshoe bats have been proposed to serve as natural reservoir hosts for SARS-CoV-2, similar to SARS-CoV and MERSCoV (Mackenzie \& Smith, 2020). Moreover, genomic analysis indicates that SARS-CoV-2 is in the same beta-CoV clade as SARS-CoV and MERS-CoV (Lu et al., 2020). In particular, SARS-CoV-2 has been observed to share almost $80 \%$ of the genome with SARS-CoV (Zhou \& Liu, 2020: Chan et al., 2020) and almost all encoded proteins of SARSCoV-2 are homologous to SARS-CoV proteins (Lu et al., 2020). In contrast, SARS-CoV-2 has been found to be more distant from MERS-CoV, with only 50\% identity ( $\mathrm{Lu}$ et al., 2020). Moreover, the entry of SARS-CoV-2 into human host cells has been found to rely on the same receptor as SARSCoV: as shown in Fig. 2, the surface angiotensin-converting enzyme 2 (ACE2), which is expressed in the type II surfactant-secreting alveolar cells of the lungs (Hoffmann et al., 2020; Wan et al., 2020). 


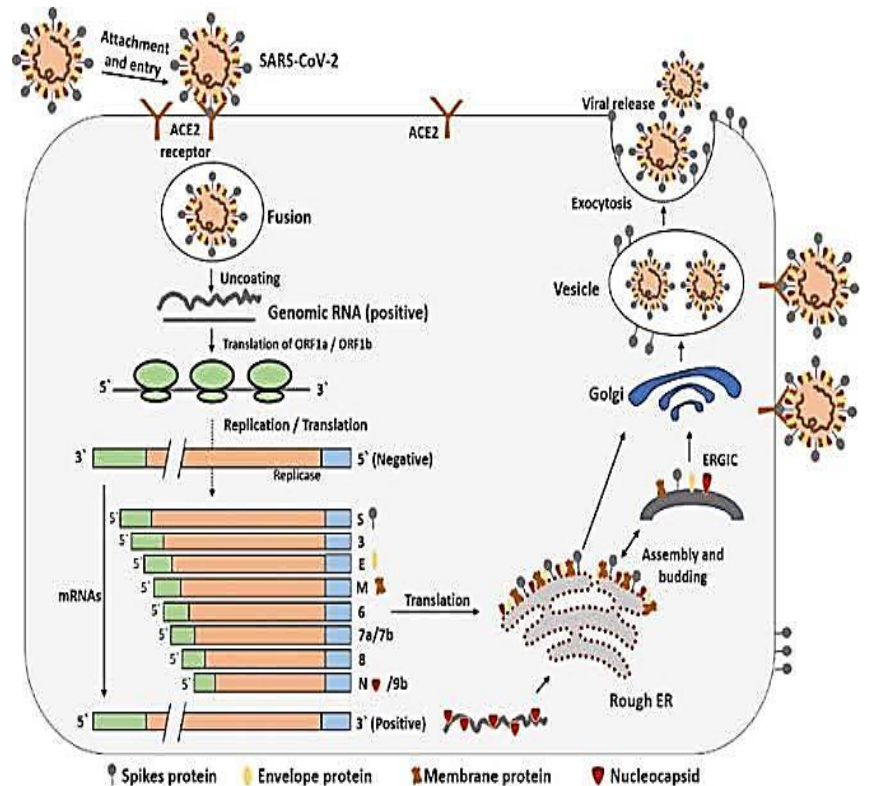

Fig. 2 Highlighting the SARS-CoV-2 receptor mediation into the cell and its onward replication

Consistently, despite amino acid variations at specific key residues, homology modeling revealed a structural similarity between the receptor-binding domains of SARS-CoV and SARS-CoV-2 (Lu et al., 2020). Consequently, the spike protein of the SARS-CoV-2 coding the genetic information gets the virus fused, uncoated, translated and replicated passing through the endoplasmic reticulum in the cell proceeding to elicit its eventual deleterious and deteriorating action. However, further studies are necessary to compare SARS-CoV and SARS-CoV-2 affinities to ACE2 receptor that might explain the increased transmissibility and greater virulence of SARS-CoV-2 compared with SARS-CoV (Hoffmann et al., 2020). Two independent groups provided key insights into the first step of SARS-CoV2 infection, by demonstrating that ACE2 host receptor is required for host cell entry of SARS-CoV-2 (Hoffmann et al., 2020; Ou, et al., 2020) Noteworthy, the expression of ACE2 receptors is not only restricted to the lung, and extra pulmonary spread of SARS-CoV in ACE-expressing tissues has been demonstrated (Ding et al., 2004). Hence the same pattern may be expected for SARS-CoV-2, with most of human tissues, such as oral mucosa and gastrointestinal tract, kidney, heart, blood vessels expressing ACE2 receptors, particularly prone to SARS-CoV2 infection (Xu et al., 2020)

\section{POSSIBLE SARS-COV-2 COUNTERACTION AT THE ENTRY STAGE}

The viral entry of SARS-CoV-2 has been further found to be prevented by a clinically proven inhibitor of the cellular host type 2 transmembrane serine protease TMPRSS2 (camostat mesylate) (Hoffmann et al., 2020). Priming of the envelopelocated trimeric spike (S) protein by host proteases, which cleave at the S1/S2 and the S2' sites, has been described as a fundamental step for viral entry, and the host protease
TMPRSS2 emerged as a key cellular factor necessary for the priming of $\mathrm{S}$ protein and for the consequent membrane fusion and viral internalization by endocytosis in the pulmonary epithelium (Hoffmann et al., 2020). Hence, TMPRSS2 has been proposed as a potential target for clinical intervention, Zhou \& Liu, (2020); Hoffmann et al., (2020) and its inhibitor camostat mesylate, approved for human use in Japan to treat pancreatic inflammation, has attracted the attention of the scientific community. Currently, a randomized, placebocontrolled, phase IIa trial is investigating the use of camostat mesylate (NCT04321096) and is expected to run until December 2020, whereas another independent trial is currently undergoing to evaluate the efficacy of camostat mesilate in combination with hydroxychloroquine in hospitalized patients with moderate COVID-19 infection. A detailed analysis of additional mechanisms of cellular viral infection for SARS-CoV-2 is still missing and would be fundamental to identify further potential biological substrates to target.

\section{IMPACT AND IMPLICATIONS OF COVID-19 ON THE HUMAN BODY: IMMUNOPATHOLOGY.}

The majority of COVID-19 cases (about 80\%) is asymptomatic or exhibits mild to moderate symptoms, but approximately the $15 \%$ progresses to severe pneumonia and about 5\% eventually develops acute respiratory distress syndrome (ARDS), septic shock and/or multiple organ failure (Huang et al., 2020; Xu, Z et al., 2020). As for SARS and MERS, the most common symptoms of COVID-19 are fever, fatigue, and respiratory symptoms, including cough, sore throat and shortness of breath (Huang. et al., 2020; Wu et al., 2020). Notably, SARS-CoV-2 infection activates innate and adaptive immune response, thus sustaining the resolution of COVID-19. While a rapid and well-coordinated immune response represents the first line of defense against viral infection, Fig. 3 portrays excessive inflammatory innate response and dysregulated adaptive host immune defense which leads to harmful tissue damage both at the site of virus entry and at systemic level.

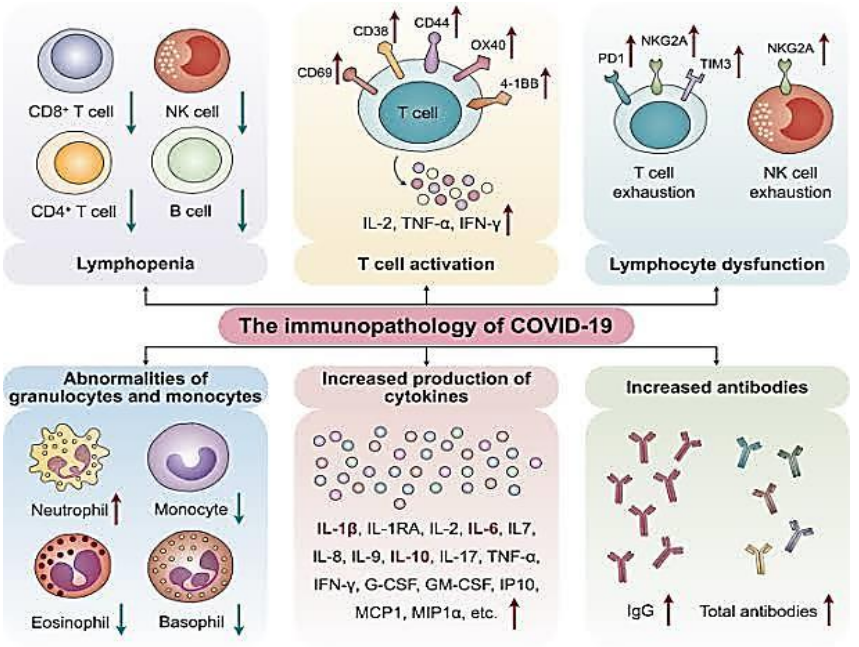

Fig. 3 A portray of the Immunopathology of Covid-19 
The excessive pro-inflammatory host response has been hypothesized to induce an immune pathology resulting in the rapid cause of acute lung injury (ALI) and ARDS occurring in SARS-CoV-2 infected patients (Huang et al., 2020; Wu et al., 2020). For example, the massive cytokine and chemokine release, the so-called "cytokine storm", clearly reflects a widespread uncontrolled dysregulation of host immune defense. Thus, given the key role of the immune system in COVID-19, a deeper understanding of the mechanism behind the immune dysregulation, as well as of SARS-CoV-2 immune-escape mechanisms might give us clues for the clinical management of the severe cases and for preventing the transition from mild to severe stages. Moreover, although no within the goal of the present review, future investigations concerning the systemic effects of uncontrolled immune system on other physiological systems, such as the gastrointestinal tract, neuroendocrine, renal and cardiovascular are urgent. Several studies highlight relevant changes occurring both in innate and adaptive immune system in COVID-19 patients. In particular, lymphocytopenia and a modulation in total neutrophils are common hallmarks and seem to be directly correlated with disease severity and death (Zhou \& Liu, 2020; Wu et al., 2020). In patients with severe COVID-19, a marked decrease in the levels of absolute number of circulatingCD4+ cells, CD8+ cells, B cells and natural killers (NK) cells, (Huang et al., (2020); Xu, Z et al., (2020); Shi et al., (2020) as well as a decrease in monocytes, eosinophils and basophils has been reported (Shi et al., 2020); (Zhang, B. et al., 2020).

In addition, most of patients with severe COVID-19 displayed significantly increased serum levels of proinflammatory cytokines (e.g. IL-6, IL-1 $\beta$, IL-2, IL-8, IL-17, G-CSF, GMCSF, IP-10, MCP-1, CCL3, and TNF $\alpha$ ) as shown in Fig. 3 (Qin et al., 2020); (Mehta et al., 2020). Although no direct evidence for pro-inflammatory cytokines and chemokines involvement in lung pathology in COVID-19 has been reported, an increase in serum cytokine and chemokine levels, as well as in neutrophil-lymphocyte-ratio (NLR) in SARSCoV-2 infected patients has been correlated with the severity of the disease and adverse outcomes, suggesting a possible role for hyper-inflammatory responses in COVID-19 pathogenesis (Qin et al., 2020). Moreover, a recent multicenter retrospective cohort study analyzing data from the Early Risk Stratification of Novel Coronavirus Pneumonia (ERS-COVID-19) study (ChiCTR2000030494) showed that patients with COVID-19 had elevated high-sensitivity Creactive protein (Hs-CRP) and procalcitonin serum levels, two major inflammation markers associated with high risks of mortality and organ injury (Li, D. et al., 2020).

\section{MECHANISM OF IMMUNE SYSTEMS IN HUMAN BODY AGAINST COVID-19}

As there is no registered medicine or vaccine against COVID19 as at August 2020, our immune system is the best defense. The immunity system supports our body's natural ability to defend against pathogens which include viruses, bacteria, fungi, protozoan, and worms, khanacademy.org/science, (2020) resist infections. As long as the immune system runs smoothly, we do not notice infections like COVID-19. Our immune system can be categorized into three categories. They are, namely, innate immunity (rapid response), adaptive immunity (slow response), and passive immunity. Passive immunity is again two types and they are natural immunity which we inherit genetically and artificial immunity that we receive from medicine. Skin and inflammatory response begins when our body is affected (wikibooks.org/wiki/HumanPhysiology, 2020). However, when our body encounters any germs or viruses for the first time, the immune system cannot work properly and we become sick. The same thing has happened in the case of COVID-19 (Chaussabel et al., 2010).

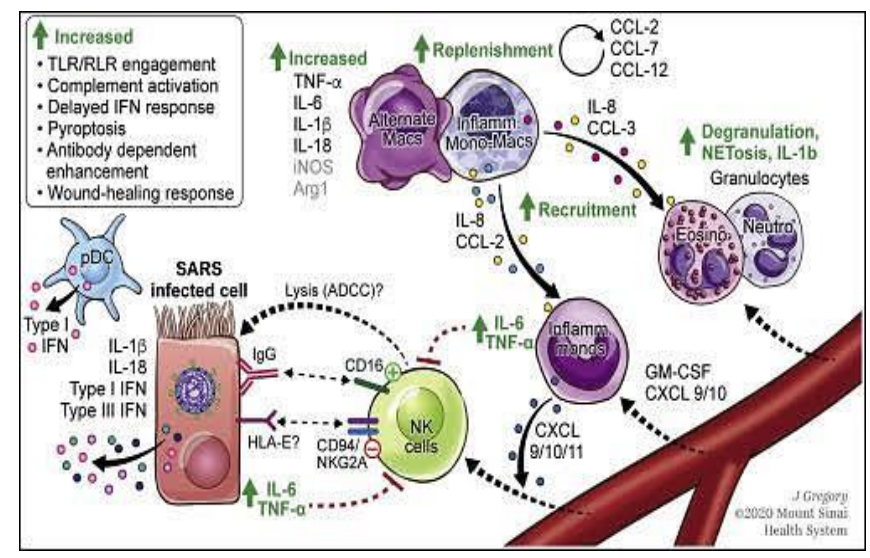

Fig. 4 Overview of the immune responses towards Covid-19

When the cells of the immune system become educated, it completes its jobs by recirculating between central and peripheral lymphoid organs and transferring it and from sites of injury via blood. Blood carries naive and educated immune cells from one site to another, as it flows throughout the body, it acts as a pipeline for the immune system. The cells again enter into the bloodstream to be transported to tissues throughout the body after exiting these nodes through outgoing lymphatic vessels (Kumar et al., 2020). Fig. 4 entails the whole highlighting strategies of the early actions of Natural Killers (NK), Interleukines (IL)'s with all their essential and functioning subunits as well as the immunoglobulins (Ig)'s. Many molecular and cellular profiling assays are now abundant for the study of the human immune system. The level of advancement of instruments has happened such as polychromatic flow cytometers have improved over the past few years. In the fields of genomics and proteomics, major technological breakthroughs have also happened, thus creating today a unique facility for the study of human beings in health and disease where inherent heterogeneity dictates that large collections of samples be analyzed (Kumar et al., 2020). After being affected by virus, immune respond to mediate anti-body. The B cells are assisted by $\mathrm{T}$ cells to differentiate into plasma cells, which in return produce antibodies specific to a viral antigen. Neutralizing nature antibody is efficient in fully blocking the 
virus from entering into host cells to limit the infection and plays a very intense protective role at the later stage of infection and prevents relapse of infection in the future. In contrast, a cellular immunity response can be seen inside the infected cells, which is mediated by T-lymphocytes. The overall adaptive immune response is directed by helper $\mathrm{T}$ cells, while cytotoxic $\mathrm{T}$ cells play a vital role in the clearance and cleaning of viral infected cells (Kumar et al., 2020).

\section{A SYNERGIC MANIPULATION OF THE RAAS SYSTEM TOWARDS ADDRESSING COVID-19}

A Synergic approach of modifying the RAAS system in COVID-19 aiming at reducing the formation of proinflammatory mediators as well as increasing the formation of anti-inflammatory mediators is focused. Counterbalance of the pro-inflammatory cascade could be achieved by strengthening the ACE2 product signaling pathways or weakening the ACE1 product signaling pathways, or both. The proinflammatory cascade may be weakened by inhibition of ACE1, preventing formation of Ang II, or by AT1-receptor antagonism/blockade of AT1-receptor, reducing the actions of Ang II at AT1-receptors and diverting Ang II to some antiinflammatory actions involving the AT2-receptors. However, since ACE1, as well as ACE2, is required for the formation of Ang (1-7), blockade of AT1-receptors is the theoretically better pharmacological option, diverting angiotensin II, III \& IV to anti-inflammatory actions, and allowing formation of the anti-inflammatory agent Ang(1-7). In contrast, ACE1 inhibition, while preventing formation of angiotensin II, also prevents formation of Ang(1-7). However, it must be noted that the overall balance between pro- and anti-inflammatory mediators achieved in the two situations (ARB and ACE1 inhibition) is not well established. Strengthening the ACE2 product signalling pathways can be achieved by increasing ACE2 levels in the plasma by recombinant enzyme, which in addition provides false targets for the virus, or by agonists mimicking products of ACE2, particularly Ang(1-7).

Plasma angiotensin II levels are reported to be higher than of normal range in the majority of COVID-19 cases (90.2\%), and were higher in critically ill COVID-19 patients than in those with mild COVID-19 symptoms (Wu et al., 2020). This may suggest that ARB's or ACE1 inhibitors may prove useful in treating COVID-19. The high plasma angiotensin II levels may have to be considered in dosing with ARB's, whereas ACE1 inhibitors directly lower angiotensin II levels, but admittedly with more side effects and with loss of some antiinflammatory mediators.

Often overlooked in the consideration of the more limited Renin Angiotensin System (RAS), is aldosterone, truly a component of the wider RAAS. Antagonists for mineralocorticoid receptors such as the aldosterone analogue spironolactone prevent actions of aldosterone including sodium retention. The combination of mineralocorticoid receptor antagonists with other antihypertensives improve blood pressure control in patients with resistant hypertension
(Morimoto \& Ichihara, 2020). Antagonists of aldosterone have also been found to increase ACE2 levels in human macrophages (Keidar et al., 2005). Aldosterone could theoretically be detrimental in COVID-19 infection by renal tubular actions to produce sodium retention, but also by tissue actions including endothelial alterations and immune system activation, resulting in pro-inflammatory actions (Davel et al., 2018).

Increase in ACE2 may theoretically be detrimental or beneficial, but this has already been discussed in Section 2 above, particularly in terms of reducing infection.

Plasma angiotensin II, but not renin, was significantly higher in critically ill COVID-19 patients than in control or those with mild COVID-19 symptoms (Wu et al., 2020). Hence, elevated plasma angiotensin II elevation in severe COVID-19 infection may be due to loss of ACE2 by binding of CoV-2 rather than to increased renin activity, and this elevated angiotensin II may be a major pathological factor in critically ill COVID-19 patients (Wu et al., 2020). Hence, restoring ACE2 or employing agonists for AT2- or masreceptors may be of potential therapeutic benefit in the later stage of infection.

Stimulation of either of the above receptors is likely to prove beneficial. Ang II acts on the AT2-receptor to produce counterbalancing anti-inflammatory actions. Ang(1-7) may act on a distinct receptor, the mas receptor to produce effects such as vasodilatation to oppose the actions of Ang II. Ang IV may also act on a specific AT-4 receptor which has been shown to have cardioprotective effect by inhibition of apoptosis (Park et al., 2016). Potential pharmacological strategies targeting the RAAS for treatment of COVID-19 have been discussed in recent publications (Zhang et al., 2020: Sriram \& Insel, 2020), although it must be cautioned that, at present, these are only of unsubstantiated theoretical benefit.

ACE inhibitors and AT1R blockers have a protective cardiovascular effect by limiting the AT1R-mediated response, but also increase the ACE2 expression enhancing the vasoprotective axis of the RAAS. Higher levels of ACE2 would also reduce the risk of developing atherosclerosis. It is not so surprising that the identification of ACE2 as the receptor for SARSCoV-2 raised speculations about the detrimental effect of RAAS-drugs in relation to COVID-19. ACEIs and ARBs promote the expression of ACE2 perhaps making the patients treated with these drugs more susceptible to infection, which is important when we consider the relationship between COVID-19, hypertension and mortality

\section{CONCLUSION}

The isolation of SARS-CoV-2 and the viral genome sequencing has thus facilitated diagnostic testing, epidemiological tracking, as well as investigations on potential preventive and therapeutic strategies in the 
management of COVID-19. To date (August, 2020), despite the intense scientific effort demonstrated by many clinical trials currently underway, no vaccines or effective drugs have been approved to prevent or treat COVID-19 meanwhile, the current standard care is supportive treatment. Therefore, based on the fast and global spread of the virus, urgent investigations are warranted in order to develop effective therapies. Within this context, treatments addressing the immunopathology of the infection have become a major focus. However, inoculation of supportive immunity as well as successive alternate inhibition of ACE1, blocking AT1 receptor, and or blocking AT2 receptor is an enhanced approach towards thwarting the SARS-Cov-2 incorporation into the system. An important consideration that needs to be done for the pathophysiology of COVID-19 is related to the ACE2 internalization mediated by SARS-COV-2 that could potentially induce a reduction of ACE2 on cell surface and then determine the absence of a key factor important for the local pulmonary synthesis of A1-7. Indeed, an imbalance between AII and A1-7 levels may exacerbate the lung injury caused by SARS-COV-2, contributing to the reduction of the pulmonary function and the increase of fibrosis and inflammation (Triassi et al., 2019).

More naturally, ingesting food of high great value of supplements in up-shooting immune system as well as ensuring physical fitness and environmental hygiene are paramount to adherence in this trying moments and indeed all the time, as the world is looking forward to unveiling the veritable vaccine and outright therapeutics against the Corona virus.

\section{REFERENCES}

[1] Bullock, G.R., Steyaert, I., Bilbe, G., Carey, R.M., Kips, J., De Paepe, J.B., Pauwels, R., Praet, M., Siragy, H.M., de Gasparo, M., 2001. Distribution of type-1 and type-2 angiotensin receptors in the normal human lung and in lungs from patients with chronic obstructive pulmonary disease. Histochem. Cell Biol. 115 (2), $117-124$.

[2] Chan, J.F.W., Lau S.K.P., To, K.K.W., Cheng, V.C.C., Woo, P.C.Y., Yuen K-Y, Middle East respiratory syndrome coronavirus: another zoonotic betacoronavirus causing SARS-like disease. Clin. Microbiol. Rev.28,465-522 (2015).

[3] Chan JF, Kok KH, Zhu Z, Chu H, To KK, Yuan S, Yuen KY. Genomic characterization of the 2019 novel human-pathogenic coronavirus isolated from a patient with atypical pneumonia after visiting Wuhan. Emerg Microbes Infect. 2020 Jan 28;9(1):221236. doi: 10.1080/22221751.2020.1719902. Erratum in: Emerg Microbes Infect. (2020)

[4] Chaussabel D, Pascual V, Banchereau J. Assessing the human immune system through blood transcriptomics. BMC Biol 2010;8:84

[5] Corman, V. M., Lienau, J. \& Witzenrath, M. Coronaviruses as the cause of respiratory infections. Internist 60, 1136-1145 (2019).

[6] De Gasparo, M., Catt, K.J., Inagami, T., Wright, J.W., Unger, T., 2000. International union of pharmacology. XXIII. The angiotensin II receptors. Pharmacol. Rev. 52 (3), 415-472.

[7] Ding, Y., He, L., Zhang, Q., Huang, Z., Che, X., Hou, J., Wang, H., Shen, H., Qiu, L., Li, Z., Geng, J., Cai, J., Han, H., Li, X., Kang, W., Weng, D., Liang, P., \& Jiang, S. (2004). Organ distribution of severe acute respiratory syndrome (SARS) associated coronavirus (SARS-CoV) in SARS patients: implications for pathogenesis and virus transmission pathways. The Journal of pathology, 203(2), 622-630. https://doi.org/10.1002/path.1560

[8] Field CJ, Johnson IR, Schley PD. Nutrients and their role in host resistance to infection. J Leukoc Biol 2002;71:16-32.

[9] Guan WJ, Ni ZY, Hu Y, Liang WH, Ou CQ, He JX, et al. Clinical Characteristics of Coronavirus Disease 2019 in China. The New England journal of medicine. 2020. https://doi.org/10.1056/ NEJMoa2002032.

[10] Guo Yan-Rong, Cao Qing-Dong, Hong Zhong-Si, Tan YuanYang, Chen Shou-Deng, Jin Hong-Jun, et al. The origin, transmission and clinical therapies on coronavirus disease 2019 (COVID-19) outbreak - an update on the status. MilMed Res 2020.

[11] Hoffmann, M., Kleine-Weber, H., Schroeder, S., Krüger, N., Herrler, T., Erichsen, S., Schiergens, T. S., Herrler, G., Wu, N. H., Nitsche, A., Müller, M. A., Drosten, C., \& Pöhlmann, S. (2020). SARS-CoV-2 Cell Entry Depends on ACE2 and TMPRSS2 and Is Blocked by a Clinically Proven Protease Inhibitor. Cell, 181(2), 271-280.e8. https://doi.org/10.1016/j.cell.2020.02.052

[12] https://en.wikibooks.org/wiki/Human Physiology/The Immune System

[13] https://www.khanacademy.org/science/hig -school-biology/hshuman-body-systems/hs-the-immune-system/a/hs-the-immunesystem-review.

[14] Huang, C., Wang, Y., Li, X., Ren, L., Zhao, J., Hu, Y., Zhang, L., Fan, G., Xu, J., Gu, X., Cheng, Z., Yu, T., Xia, J., Wei, Y., Wu, W., Xie, X., Yin, W., Li, H., Liu, M., Xiao, Y., ... Cao, B. (2020). Clinical features of patients infected with 2019 novel coronavirus in Wuhan, China. Lancet (London, England), 395(10223), 497506. https://doi.org/10.1016/S0140-6736(20)30183-5

[15] Jayaweera J, Reyes M, Joseph A. Childhood iron deficiency anemia leads to recurrent respiratory tract infections and gastroenteritis. Sci Rep 2019;9:12637. Maares M, Haase H. Zinc and immunity: an essential interrelation. Arch Biochem Biophys 2016;611:58-65.

[16] Jin YH, Cai L, Cheng ZS, Cheng H, Deng T, Fan YP, et al. A rapid advice guide-line for the diagnosis and treatment of 2019 novel coronavirus (2019-nCoV) infected pneumonia (standard version). Mil Med Res 2020;7:4.

[17] Kumar S, Nyodu R, Maurya VK, Saxena SK. Host Immune Response and Immunobiology of Human SARS-CoV-2 Infection. Coronavirus Disease 2019 (COVID-19). 2020;43-53. Published 2020 Apr 30. doi:10.1007/978-981-15-4814-7_5

[18] Kwok KO, Lai F, Wei WI, Wong SYS, Tang JWT. Herd immunity - estimating the level required to halt the COVID-19 epidemics in affected countries. JInfect 2020.

[19] Li Q, Guan X, Wu P, et al. Early transmission dynamics in Wuhan, China, of novel coronavirus infected pneumonia. N Engl $\mathbf{J}$ Med 2020;19.

[20] $\mathrm{Li}$, D. et al. Immune dysfunction leads to mortality and organ injury in patients with COVID-19 in China: insights from ERSCOVID-19 study. Signal Transduct. Target. Ther. 5, 62 (2020).

[21] Lu, R., Zhao, X., Li, J., Niu, P., Yang, B., Wu, H., Wang, W., Song, H., Huang, B., Zhu, N., Bi, Y., Ma, X., Zhan, F., Wang, L., Hu, T., Zhou, H., Hu, Z., Zhou, W., Zhao, L., Chen, J., Tan, W. (2020). Genomic characterisation and epidemiology of 2019 novel coronavirus: implications for virus origins and receptor binding. Lancet (London, England), 395(10224), 565-574. https://doi.org/10.1016/S0140-6736(20)30251-8

[22] Mackenzie, J. S. \& Smith, D. W. COVID-19: a novel zoonotic disease caused by a coronavirus from China: what we know and what we don't. Microbiol. Aust. https://doi.org/10.1071/MA20013 (2020).

[23] Mehta, P., McAuley, D. F., Brown, M., Sanchez, E., Tattersall, R. S., Manson, J. J., \& HLH Across Speciality Collaboration, UK (2020). COVID-19: consider cytokine storm syndromes and immunosuppression. Lancet (London, England), 395(10229), 1033-1034. https://doi.org/10.1016/S0140-6736(20)30628-0

[24] Meszaros L. How to boost your immune system during the COVID-19 pan-demic. Intern Med (2020). 
[25] Nonnecke BJ, McGill JL, Ridpath JF, Sacco RE, Lippolis JD, Reinhardt TA. Acute phase response elicited by experimental bovine diarrhea virus (BVDV) infec-tion is associated with decreased vitamin D and E status of vitamin-replete preruminant calves. J Dairy Sci 2014;97:5566-79.

[26] Norman K, Pichard C, Lochs H, Pirlich M. Prognostic impact of disease-related malnutrition. Clin Nutr 2008;27:5-15.

[27] Ou, X., Liu, Y., Lei, X., Li, P., Mi, D., Ren, L., Guo, L., Guo, R., Chen, T., Hu, J., Xiang, Z., Mu, Z., Chen, X., Chen, J., Hu, K., Jin, Q., Wang, J., \& Qian, Z. (2020). Characterization of spike glycoprotein of SARS-CoV-2 on virus entry and its immune crossreactivity with SARS-CoV. Nature communications, 11(1), 1620. https://doi.org/10.1038/s41467-020-15562-9

[28] Prompetchara Eakachai, Ketloy Chutitorn, Palaga Tanapat. Immune responses in COVID-19 and potential vaccines: lessons learned from SARS and MERSepidemic. Asian Pac J Allergy Immunol 2020

[29] Qin, C., Zhou, L., Hu, Z., Zhang, S., Yang, S., Tao, Y., Xie, C., Ma, K., Shang, K., Wang, W., \& Tian, D. S. (2020). Dysregulation of Immune Response in Patients With Coronavirus 2019 (COVID19) in Wuhan, China. Clinical infectious diseases : an official publication of the Infectious Diseases Society of America, 71(15), 762-768. https://doi.org/10.1093/cid/ciaa248

[30] Rothe C, Schunk M, Sothmann P, Bretzel G, Froeschl G, Wallrauch C, et al. Transmission of 2019-nCoV Infection from an Asymptomatic Contact in Germany. The New England journal of medicine. 2020;382:970-1. https://doi.org/10.1056/NEJMc2001468.

[31] Schuetz P, Fehr R, Baechli V, Geiser M, Deiss M, Gomes F, Individualised nutritional support in medical inpatients at nutritional risk: a randomised clinical trial. Lancet 2019;393:2312-21.

[32] Shi, Y., Tan, M., Chen, X., Liu, Y., Huang, J., Ou, J., Deng, X., Immunopathological characteristics of coronavirus disease 2019 cases in Guangzhou, China. medRxiv https://doi.org/10.1101/2020.03.12.20034736 (2020).

[33] Sriram, K., Insel, P.A., 2020. A hypothesis for pathobiology and treatment of COVID-19: the centrality of ACE1/ACE2 imbalance. Br. J. Pharmacol. https://doi.org/10.1111/bph.15082 (Epub ahead of print).

[34] Triassi M., Nardone A., Giovinetti M. C., De Rosa E., Canzanella S., Sarnacchiaro P., et al. (2019). Ecological Risk and Estimates of Organophosphate Pesticides Loads into the Central Mediterranean Sea from Volturno River, the River of the "Land of Fires" Area, Southern Italy. Sci. Total Environ. 678, 741-754. 10.1016
[35] Walls, A.C., Park, Y.-J., Tortorici, M.A., Wall, A., McGuire, A.T., Veesler, D., 2020. Structure, function, and antigenicity of the SARS-CoV-2 spike glycoprotein. Cell 181, 281-292.

[36] Wan, Y., Shang, J., Graham, R., Baric, R. S. \& Li, F. Receptor recognition by the novel coronavirus from Wuhan: an analysis based on decade-long structural studies of SARS coronavirus. J. Virol. 94, e00127-20 (2020).

[37] Wang D, Hu B, Hu C, Zhu F, Liu X, Zhang J. Clinical Characteristics of 138 Hospitalized Patients With 2019 Novel Coronavirus-Infected Pneumonia in Wuhan, China. Jama. 2020. https://doi.org/10.1001/jama.2020.1585.

[38] Wessling-Resnick M. (2018). Crossing the Iron Gate: Why and How Transferrin Receptors Mediate Viral Entry. Annual review of nutrition, 38, 431-458. https://doi.org/10.1146/annurev-nutr082117-051749

[39] Wu, F., Zhao, S., Yu, B., Chen, Y. M., Wang, W., Song, Z. G., Hu, Y., Tao, Z. W., Tian, J. H., Pei, Y. Y., Yuan, M. L., Zhang, Y. L., Dai, F. H., Liu, Y., Wang, Q. M., Zheng, J. J., Xu, L., Holmes, E. C., \& Zhang, Y. Z. (2020). A new coronavirus associated with human respiratory disease in China. Nature, 579(7798), 265-269. https://doi.org/10.1038/s41586-020-2008-3

[40] Xu, H., Zhong, L., Deng, J., Peng, J., Dan, H., Zeng, X., Li, T., \& Chen, Q. (2020). High expression of ACE2 receptor of 2019$\mathrm{nCoV}$ on the epithelial cells of oral mucosa. International journal of oral science, 12(1), 8. https://doi.org/10.1038/s41368-020$\underline{0074-\mathrm{x}}$

[41] Xu, Z., Shi, L., Wang, Y., Zhang, J., Huang, L., Zhang, C., Liu, S., Zhao, P., Liu, H., Zhu, L., Tai, Y., Bai, C., Gao, T., Song, J., Xia, P., Dong, J., Zhao, J., \& Wang, F. S. (2020). Pathological findings of COVID-19 associated with acute respiratory distress syndrome. The Lancet. Respiratory medicine, 8(4), 420-422. https://doi.org/10.1016/S2213-2600(20)30076-X

[42] Zhang, B., Zhou, X., Zhu, C., Song, Y., Feng, F., Qiu, Y., Feng, J., Jia, Q., Song, Q., Zhu, B., \& Wang, J. (2020). Immune Phenotyping Based on the Neutrophil-to-Lymphocyte Ratio and IgG Level Predicts Disease Severity and Outcome for Patients With COVID-19. Frontiers in molecular biosciences, 7, 157. https://doi.org/10.3389/fmolb.2020.00157

[43] Zhang, H., Penninger, J.M., Li, Y., Zhong, N., Slutsky, A.S., 2020. Angiotensinconverting enzyme 2 (ACE2) as a SARS-CoV-2 receptor: molecular mechanisms and potential therapeutic target. Intensive Care Med. 46, 586-590.

[44] Zhou, L., Liu, K. \& Liu, H. G. Cause analysis and treatment strategies of recurrence' with novel coronavirus pneumonia (covid19) patients after discharge from hospital. Zhonghua Jie $\mathrm{He} \mathrm{He} \mathrm{Hu}$ Xi Za Zhi Zhonghua Jiehe He Huxi Zazhi Chin. J. Tuberc. Respir. Dis. 43, E028 (2020). 